\title{
From Mexico to Michigan and back: An international collaboration investigating primate behavior, ecology, and evolution from multiple perspectives
}

\author{
Liliana Cortés-Ortiz $^{1}$ (1) | Domingo Canales Espinosa ${ }^{2}$ | Pedro D. Dias ${ }^{2}$ | \\ Thore Bergman $^{3}$ | Dawn M. Kitchen ${ }^{4,5}$
}

${ }^{1}$ Department of Ecology and Evolutionary Biology, University of Michigan, $1105 \mathrm{~N}$. University, Ann Arbor, Michigan

${ }^{2}$ Primate Behavioral Ecology Lab, Instituto de Neuroetología, Universidad Veracruzana, Xalapa, Veracruz, Mexico

${ }^{3}$ Department of Psychology, University of Michigan, Ann Arbor, Michigan

${ }^{4}$ Department of Anthropology, The Ohio State University, Mansfield, Ohio

${ }^{5}$ Department of Anthropology, The Ohio State University, Columbus, Ohio

\section{Correspondence}

Liliana Cortés-Ortiz, Department of Ecology and Evolutionary Biology, University of Michigan, 1105 N. University, Ann Arbor, MI 48109.

Email: Icortes@umich.edu

Funding information

National Science Foundation, Grant/Award Numbers: BCS-0962807, BCS-0962755; University of Michigan OVPR-Faculty Grant; The Ohio State University; Universidad Veracruzana

\begin{abstract}
Evolutionary research benefits form the integration of laboratory and field components to determine factors and processes that affect the evolutionary trajectories of species. Our shared interest in understanding hybridization with genetic admixture as a process that may impact social, behavioral, and ecological features of primates, brought us together in a collaborative project aimed at addressing how vocal variation in two species of howler monkeys in Mexico affects and is affected by hybridization. To achieve this goal, we joined our academic expertise in studying primate genetics, ecology, and behavior under different natural and experimental conditions. We took advantage of decades of experience studying and handing wild howler monkeys for translocation projects to safely sample and study wild populations for this project. Here, we describe the history of our collaboration highlighting how our different perspectives, academic realities, and individual strengths built the foundation for our successful collaboration. We also share our perspectives on how this collaboration opened up new academic venues, broadened our individual perspectives on the integration of different research approaches to address a complex topic, and allowed us to recognize the strength of international collaboration.
\end{abstract}

KEYWORDS

Alouatta palliata, Alouatta pigra, genetics, hybridization, vocalization
Primates live in complex ecological and social environments that are different among distinct lineages. These social differences arise as lineages become separated, begin to diverge, and eventually speciate. Current evidence shows that evolution does not always occur through clean bifurcating events, in which an ancestor splits into two (or more) forms that diverge and form new lineages incapable of interbreeding. Rather, diversification often includes episodes of interactions between divergent lineages, in which exchange of genetic material through hybridization may affect their evolutionary trajectories (Arnold, 2006; Grant \& Grant, 1992; Mallet, 2005). Hybridization is now recognized as an important evolutionary process in human (Green et al., 2010; Reich et al., 2010; Sankararaman et al., 2014) and nonhuman primates (Arnold \& Meyer, 2006; Cortés-Ortiz, Roos, \& Zinner, 2019; Zinner, Arnold, \& Roos, 2011). Our collaboration has its origin in our shared interest in understanding how different biological (ecological, genetic) and social (behavior, communication) traits of primates affect and are affected by hybridization. This complex question requires an understanding of species as a whole, with particular social complexities existing under one or more environmental regimes. Furthermore, it requires an understanding of the genetic variation that may enhance or constrain the evolutionary potential of each species. We address this broad 
question by studying a natural hybrid zone between two species of howler monkeys (the mantled howler monkey, A. palliata, and the black howler monkey, A. pigra) in Tabasco, Mexico.

The study of the howler monkey hybrid zone in southern Mexico started as part of the Ph.D. research of the first author (Liliana Cortés-Ortiz, hereafter Liliana). She had previously studied the reproductive behavior of a group of mantled howler monkeys as part of a team lead by Ernesto Rodríguez-Luna at the Universidad Veracruzana (UV). Members of this team studied the ecology and behavior of mantled howler monkeys and were involved in numerous projects related to the conservation of Mexican primates. In the late 1990 s, Rodríguez-Luna planned expeditions to Tabasco in search of a previously described area of sympatry (Smith, 1970) between mantled howler monkeys and black howler monkeys (A. pigra) with the intention of setting up a field site where both species could be studied under similar environmental conditions. Domingo Canales Espinosa (hereafter Domingo) was also part of that research team and together with Francisco García Orduña (also from UV) and the late Joaquim Veà (Universitat de Barcelona, Spain) traveled to Tabasco where they found groups of the two howler monkey species living in close proximity and groups with individuals of both species living together. The level of morphological variation of individuals in the area, as well as the distinct vocalizations of some individuals, provided the foundation to suspect that hybridization between these species could be occurring. Liliana became highly interested in this finding and, as part of her Ph. D. research, designed a genetic study to determine whether hybridization was indeed occurring between these species. The result was the first genetic evidence of hybridization of Neotropical primates (Cortés-Ortiz et al., 2007).

\section{1 | OUR COLLABORATION}

In 2009, the present authors launched a collaborative project to understand the causes and consequences of vocal variation in the howler monkey hybrid zone, through an integrative approach analyzing behavior, morphology, and genetics. This is how each of us recalls how we became involved in this collaboration.

\section{1 | Liliana}

I had been studying the genetic (Cortés-Ortiz et al., 2007) and morphological (Kelaita \& Cortés-Ortiz, 2013) signatures of hybridization of howler monkeys in Mexico since 1998. This work required sampling several groups of wild howler monkeys, which was possible only because of the extensive expertise of a core field team that included veterinarians, field workers, and scientists who had worked together for several years (including the time when Liliana was a professor at UV). The genetic analyses were also enhanced by samples collected through other projects lead by Domingo (veterinarian and professor at the UV) that funded numerous field expeditions in Tabasco, Veracruz, and Campeche. Domingo had collaborated on this project since the beginning, and Pedro D. Dias (hereafter Pedro), a behavioral ecologist studying howler monkey ecology and behavior (e.g., Arroyo-Rodríguez \& Dias, 2010) became part of our collaborative team in 2005 while he was working with Domingo on a black howler monkey translocation project in Campeche. As I moved to the University of Michigan (UM) in 2004, we continued collaborating in studying the howler monkey hybrid zone. Soon after my arrival at UM, I met Thore J. Bergman (hereafter Thore), who joined the Department of Psychology at UM and who had studied the behavior of a hybrid baboon population (Bergman \& Beehner, 2004) in Ethiopia. In 2006, Dawn M. Kitchen (hereafter Dawn), who had studied howler monkey vocalizations for her $\mathrm{Ph}$. D. dissertation (e.g., Kitchen, 2004) and was faculty at the Ohio State University (OSU), visited UM, and during a brief meeting we discussed the possibility of combining our expertise to study vocal variation in hybrid howler monkeys. Three years later, Thore, Dawn, and I secured funding to launch this collaborative project.

\section{2 | Domingo}

This particular collaboration started as an extension of a long-term collaboration with Liliana that commenced when we were graduate students at the Universidad Veracruzana. Since 1989, I had been involved in translocations of Mexican primates as part of a number of conservation-related projects at UV. My previous experience handling wild howler monkeys was applied to sample wild populations of howler monkeys for this and previous projects in the hybrid zone. The howler monkey hybrid system is unique, not only for the novelty of studying hybridization but also because it involves two species for which a great deal of behavioral and ecological information is available for comparison. These conditions, together with the exceptional possibility of obtaining hardto-collect biological samples, provided the means to address questions that could not be easily tested otherwise in the wild. Therefore, I was very excited to join this collaboration with Pedro, Thore, and Dawn, when Liliana asked to continue our collaboration studying the howler monkey hybridzone.

\section{3 | Pedro}

My timeline for this collaboration begins in 2005 when I started working with Domingo, who was already Liliana's long-term collaborator. During the capturing of black howler monkeys as part of a translocation project in Campeche, I noticed that only very basic dental information was collected, so, in addition to in vivo teeth measurements, I started working on a technique for obtaining casts that could be used to collect morphological information. This interest in teeth morphology brought me closer to Liliana, and we started discussing the possibility of working together on a teeth-related project, which lead to a first formal collaboration that culminated in a series of papers with Liliana's former student, Mary Kelaita 
(e.g., Kelaita, Dias, Aguilar-Cucurachi, Canales-Espinosa, CortésOrtiz, 2011). When Liliana invited me to participate in the collaborative project on vocal variation in the hybrid zone, I felt it was a continuation of that ongoing collaboration.

\section{4 | Thore}

I do not remember exactly how we entered into this collaboration! I think I may have approached Liliana (who already worked with Pedro and Domingo) because I knew we shared an interest in primate hybrid zones. I had also previously worked with Dawn and I knew that Dawn had done her dissertation work with howler vocalizations. So, it just seemed to make sense for all of us to work together. Liliana and I got some funding from UM to conduct initial surveys and then, together with Dawn as co-PI, Liliana and I applied for a National Science Foundation grant in which Domingo and Pedro were collaborators. The main idea for the grant was to study the most salient feature of howler monkeys (their loud vocalizations) in the context of the hybrid zone. We asked two basic questions: How does genetic (or social) admixture affect their vocalizations? How do vocalizations contribute to the hybrid zone dynamics (e.g., are the vocalizations of one species more effective than the other)?

\section{5 | Dawn}

My recollection is that I was visiting Thore Bergman and Jacinta Beehner at UM (I work at OSU). Thore was excited to introduce me to someone they had met who studied howler monkeys, which had been the focus of my dissertation research (e.g., Kitchen, 2004). I was instantly mesmerized listening to Liliana describe her fascinating project in the hybrid zone. I think somewhere along the way she invited me to join her and work on the vocal aspect of the project. I think at this point she knew that Thore had worked on hybrid baboons, and so we felt it was natural for all three of us to collaborate. Then, she took me down to Mexico where I met Pedro and Domingo, and they showed me many field sites in the range of the three different populations we were interested in studying $-A$. palliata, hybrids, and A. pigra. I recall the first time I heard the hybrids. I was very familiar with the loud calls of both black and mantled howlers, which are completely distinct. When I heard my first hybrid, and before we had genetic data for that individual to confirm it was indeed a hybrid, I was blown away about how the call sounded exactly like a cross between the two. It started in like it was going to be the loud, long, low frequency, and very impressive roar of the black howler monkey and then quite suddenly became a multi-syllable version echoing the pattern of mantled howler roars. Later, this observation would lead us to experimenting to create our own versions of these calls, mixing the frequency and temporal patterns to the two, to try to identify which species-specific features, if any, the two species focused on when hearing a nearby caller.

\section{2 | COLLABORATION WORKS WHEN EVERYONE HAS A GENUINE INTEREST AND AN IMPORTANT ROLE TO FILL}

The scope of this project would not have been possible without a group that had such different interests and backgrounds. Scientific specialization allows a deeper understanding of a topic, but time limitations can prevent researchers from acquiring a deeper understanding of multiple topics in a reasonable timeframe. Our combined expertise in genetics, evolutionary biology, behavioral ecology, vocal communication, and conservation allowed the integration of a unique and feasible project. Furthermore, the fact that we all had multiple fieldwork experiences, both in terms of taxonomic breadth and in the varieties of conditions, geographical locations, and cultural idiosyncrasies under which research was conducted, allowed us to value equally everyone's contribution and make this project a reality. Since we all had worked with wild primate populations, we had a clear understanding of the importance of valuing different fieldwork experiences (what has worked and what has not) to design a feasible and effective study. Furthermore, we all had included laboratory analyses in our research programs, so we valued the precision and strength that laboratory analyses add to address particular research questions when combined with fieldwork. As a result, we integrated fieldwork and laboratory components of the study, pairing vocal and behavioral data with genetic analyses of biological samples, to address our research questions.

Besides the value of composing a collaborative team with different scientific backgrounds, the combination of researchers living in Mexico, where the study was taking place, and some living in the United States eased the implementation and funding of the project. For example, the in-country researchers already had knowledge of where and how to request the proper permits to conduct the type of work needed for the project, and provided administrative and logistic support for each stage of the project, infrastructure, institutional endorsement that facilitated paperwork, and credibility both with governmental institutions and the local communities, and funding that would otherwise be unavailable for foreign researchers. They also had trained field assistants who were invited to work on the project and who trained new personnel. Similarly, researchers living in the US had the possibility of applying for funding to sustain a project of this magnitude, and they had access to laboratories and other infrastructure with technology that would be more costly or difficult to find in Mexico.

Previous studies on different aspects of hybridization of howler monkeys by Liliana, Domingo, and Pedro provided the framework for the study system and laid the groundwork for setting up the study of both parental (A. palliata in Veracruz and A. pigra in Campeche) and hybrid (Tabasco) populations. Furthermore, the genetic work of Liliana and her former students provided the foundation upon which to analyze the levels of admixture of individuals sampled in this study and technical expertise to sample, analyze and interpret the results of the genetic component of the project. The ample experience in studying and analyzing howler monkey (Pedro) and African papionid 
(Thore and Dawn) social behavior under natural conditions allowed the adequate methodological design for data collection in the three settings (i.e., the hybrid zone and the allopatric ranges of parental species). In particular, the use of an ethogram previously compiled and effectively used by Pedro's team to study both species of howler monkeys ensured proper comparisons between species. Dawn's expertize on black howler monkey vocalizations, as part of her Ph.D. research in Belize, together with her and Thore's expertize in studying vocal communication in African primates, permitted the selection of adequate equipment to record vocalizations and to conduct playback experiments in the field, and the analytical tools to study vocal data. Each collaborator participated in and collected data for different components of the project, but we all participated in the integration and interpretation of results.

\section{3 | PROJECT OUTCOMES}

Our collaborative project has produced several publications to date. We first characterized the loud vocalizations of male howler monkeys to identify the extent of vocal divergence between species, test predictions on the relationship between morphology (body size) and acoustics, and explore the potential role of sexual selection on the evolution of vocal variation between closely related species (Bergman et al., 2016). We found significant differences in acoustic and temporal features of the roar vocalizations of the two species of howler monkeys in Mexico, with A. pigra showing a lower fundamental frequency and smaller formant dispersion than $A$. palliata, as predicted by the body size. The characteristics of calling bouts support the notion that $A$. pigra's bouts might be more successful at intimidating rivals than $A$. palliata's, a prediction on the basis of differences in their social systems. We also compared vocal variation within and between species and their hybrids to assess the extent of vocal plasticity and genetic ancestry in determining differences in acoustic and temporal features of the loud calls (Kitchen et al., 2019). By using both field observations/recording and laboratory analysis of genetic admixture of individuals from the hybrid zone, we determined that acoustic features of vocalizations were strongly associated with genetic ancestry, but temporal features of the vocalizations showed evidence of plasticity. Finally, we explored the effect of vocal differences in recognizing potential same-sex rivals in the context of hybridization by conducting playback experiments with naïve (allopatric) populations of each species, and found specific differences in the response to heterospecific callers (Kitchen, Cortés-Ortiz, Dias, Canales-Espinosa, \& Bergman, 2018). Specifically, A. palliata males responded equally to calls from both species, but $A$. pigra only responded to conspecific calls, suggesting that $A$. pigra males may not be able to recognize interspecific rivals that could compete for reproductive opportunities.

Moreover, blood sampling allowed accurate characterization of the genetic composition of each individual in the hybrid zone, and permitted us to determine differential patterns of introgression of mitochondrial and nuclear genomes, as well as between autosomes and sex chromosomes (Cortés-Ortiz et al., 2019). We detected a bias in mitochondrial DNA introgression, a lack of introgression of the $Y$ chromosome and limited introgression of the $\mathrm{X}$-chromosome. These patterns imply that females with $A$. pigra mitochondrial DNA are more likely to hybridize or produce fertile female offspring, that males are not viable or fertile in early generations of hybridization, and that genes on the $\mathrm{X}$-chromosome may be involved in reproductive isolation as observed in other hybrid systems.

This project also allowed us to conduct the first comparative analyses of social behavior on the two howler monkey species using identical methods for data collection, and determine how specific differences in some aspects of their social structures (i.e, proximity, female affiliative behavior, etc.) affect and are affected by hybridization (Ho et al., 2014). By analyzing intraspecific variation in allopatric and sympatric populations for each species, we confirmed previously reported species differences and asserted that these differences were not the product of methodological or environmental differences. Behavioral comparisons of admixed and unadmixed individuals in sympatry showed that specific differences were paralleled by differences between pigra-like and palliata-like hybrids, even after controlling for ecological variation, implying an important genetic component in their social behavior.

Each article was led by a different team member according to area of expertise, but we all contributed to each of them-we asked the right questions, gave insights, and provided true help to one another with the goal of improving each paper. Our different fields of study and the wealth of samples and data collected through this collaboration have already fueled new projects, and we will continue working as a team to complete further analyses on the behavioral and genetic factors that affect the natural hybridization of howler monkeys in Mexico. The impact of this collaboration goes beyond scientific findings. This project allowed us to train nine Mexican students and field assistants and four US graduate students (three from OSU and one from UM) in field techniques for data collection and analyses. Furthermore, with the participation of a Mexican student (María del Socorro Aguilar Cucurachi, now a Ph.D. in education) we were able to give back to the local communities living near the hybrid zone, by providing the opportunity to elementary school children to broaden their academic experience as we implemented a conservation education workshop on the ecological importance of howler monkeys that live in close proximity to their communities. This was the first of several other workshops that María del Socorro conducted in Tabasco as part of her Ph.D. work, with the aim of generating awareness on primate conservation in the region.

\section{I WHAT DID WE LEARN FROM WORKING WITH EACH OTHER IN THIS PROJECT?}

\section{1 | Liliana}

This collaboration made me aware of the importance of understanding the different idiosyncrasies and expectations of researchers 
and students living in different countries. Having grown up and worked for several years in Mexico makes me aware of the way in which things work there and appreciate the (often unnoted and heroic) effort of my in-country colleagues to "make things happen" under, sometimes, less than ideal circumstances. Living and working in the US for many years now also allows me to recognize the effort that it takes to coordinate and conduct field work internationally, and understand the differences in the Mexican and US academic systems with their own strengths and weaknesses. Having this dual perspective allowed me to make the most of the strengths of the "two worlds" and the different abilities and expertize of the people involved in the project, recognize what is taken for granted by colleagues and students in each country, and bring together the different assets of each collaborator for the benefit of the project. My collaborators' expertise in behavioral ecology and my understanding of the hybridization process in howler monkeys allowed us to use our hybrid system to rigorously test aspects of vocal communication that are not possible to do in other settings. Pedro's and Domingo's knowledge of the behavior and ecology of the two species of howler monkeys and their extensive field experience in Mexico permitted adapting the observational and experimental design used by Thore and Dawn in studies of baboon communication. Similarly, the analytical tools used by Thore and Dawn to examine and interpret behavioral and vocal data were critical for the success of the project. My role as the link between the Mexican and US teams (researchers, students, and field assistants) allowed me to maintain fluid communication and provided the necessary context to each member of the team. I think this possibility of bridging the communication between local and foreign members of the team resulted in a positive experience that allowed everyone to value the importance of the participation of each other. Scientifically, the project gave me the opportunity to experience different ways of studying primates and challenged me to think more broadly on how to apply the genetic knowledge generated in my lab to different questions in behavioral ecology and evolution. This collaborative project surely helped me to recognize my own strengths and weaknesses, and I believe has helped me to become a better scientist.

\section{2 | Domingo}

My current position as an academic administrator leaves me little time to focus on research. This collaborative project allowed me to engage in howler monkey research in the hybrid zone, which continues to be a topic of great interest to me. It strengthened my collaboration with Pedro and Liliana, and propelled me to explore new collaborative horizons with Thore and Dawn. The financial and logistic support from the team that I lead at UV permitted the effective implementation of the project. The integration of colleagues and students from UV, UM, and OSU in all aspects of the project allowed extensive training for Mexican students and field assistants, improving local capacity and experience to continue our own research projects.

\section{3 | Pedro}

Despite previous experience studying ecology and behavior of natural primate populations, I had never worked in a project that had a field-experiment design, so I really enjoyed learning all the procedures associated with preparing and implementing the vocalization experiments. The collaboration also led me to novel and highly diverse research paths, which I had not previously considered, including genetics, morphometrics, and stable isotope analysis; and it has been a great experience! From the project's results per se, I am fascinated by the correlations between behavior and genetics in the unadmixed-to-hybrid continuum and by the amazing amount of new research questions that emerged.

\section{4 | Thore}

In general, I came to appreciate the complexity of the situation through my collaborators. I had previously worked on a baboon hybrid zone that was linear (along a river) and symmetric. I was also used to recording behavior in large troops of terrestrial monkeys where a million things are happening simultaneously and the trick was to focus on the critical interactions and ignore some of the subtleties. The howler situation was quite different. From Domingo and Pedro I came to see the patchy, mosaic nature of the howler hybrid zone and to think about how habitat variation and disturbance plays such a strong role for these arboreal monkeys. I also learned from Pedro that you really have to pay attention to the subtle behaviors of howlers (often the only behaviors they exhibit for hours on end!). From Liliana, I came to see that the genetics of the hybrid zone are extremely complicated with bimodally distributed variation (few intermediate or F1 hybrids) but also evidence of backcrossing in even "pure" looking individuals. The more genetic information you have, the messier (but more accurate and interesting) the picture will be. Finally, working with Dawn taught me that, no matter how challenging, doing playback experiments in the tropics on arboreal monkeys can be quite fun.

\section{5 | Dawn}

Liliana is the laboratory scientist, and her personality matches that - careful, patient, and precise. I am the opposite of that! However, it felt like our combination really worked to get so much done in the field and with training the students, and so forth. My understanding of the complex nature of the genetics is still rusty, but Liliana taught me more than I would have ever learned about genetics on my own. My training would suggest that I was familiar with the fine-tuned behavioral approach Pedro used given I had done detailed behavioral work with baboons (e.g., Kitchen, Seyfarth, Fischer, \& Cheney, 2003). However, howler monkeys are clearly not baboons. So, my approach to the behaviors of howler monkeys had been very coarse and dismissive. I would have never appreciated the subtleties and complexities of the three populations (A. palliata in Veracruz, 
A. pigra in Campeche and admixed individuals in the hybrid zone) had he not trained me (along with our student assistants) to follow a consistent and thorough pattern of daily behavioral collection, paying close attention to quiet vocalizations and minor movements. To be fair, this kind of approach to both individual and group would be impossible with a wild and enormous baboon group, in which each move is so obvious and large scale, but was once I caught on - not only achievable with howlers, but far more insightful and provided more details about their lives than the generalized approach I had taken during my graduate studies of howlers in Belize. On the other hand, our playback experimental approach was new to Pedro. Finally, I think my writing skills are greatly improved whenever I work with Thore. Whereas I have a tendency (as shown here) to say too much and wind around a topic, he prefers brevity and a clean, outlined style. After stewing over his cuts (sometimes it takes a few days to let go of three paragraphs that could be said in one sentence), I was always happy that he did not hold back in his editing.

\section{5 | CONCLUDING REMARKS}

Individually, we have different experiences and skills, and use particular approaches to investigate research questions on our academic fields (behavioral ecology, genetics, evolution, cognition), but together we integrated our perspectives and expertise to understand a single evolutionary phenomenon considering proximate (e.g., effect of body size or genetic makeup on vocal variation) and ultimate (e.g., the effect of intrasexual selection on male response to interspecific vocalizations) factors. Our team not only has the academic expertise to conduct the distinct research components of the project but also the understanding of the value of local and foreign perspectives to evaluate what is feasible to address and achieve as a team. Our different, but complementary outlooks on the scientific process are inherent to our different cultural backgrounds, academic training, working experiences, and day-to-day realities. Living in two countries with very distinct academic systems allows us to get the best of each of them. Finally, the shared enthusiasm and respect for the contribution of each collaborator were likely a key factor for the successful completion of this project. We hope that this commentary helps to illustrate how having a successful collaboration goes beyond adding the scientific expertise of different researchers: Respecting and capitalizing on individual strengths, skills, opportunities, and differences are cornerstones to collaborative research.

\section{ACKNOWLEDGMENTS}

We would like to thank Marilyn Norconk for her kind invitation to write this contribution. We also thank Javier Hermida, María del Socorro Aguilar Cucurachi, Antonio Jauregui, Antonio Jauregui Jr., Ariadna Rangel, Alba Rodas, María de Jesús Rovirosa, Paulo Quintana, Marcella Baiz, who assisted in the expedition to sample primates. A number of graduate and undergraduate students participated in collecting data in the field and analyzing data in the lab: Dara Adams, Antoinette Bode-Higgerson, Lucy Ho, Rosario Candelero, Rueda, Alejandro Coyohua, Roxann Chicalo, Pamela Cruz Mirós, Jason Ferrell, Alfredo Gómez Martínez, Suzanna Hammond, Ana Peralta, Lucy Redfern Cummins, Cynthia Rodríguez Maldonado, and Jay Schwartz. The study was funded by the National Science Foundation (BCS-0962807 and BCS-0962755), University of Michigan OVPR-Faculty Grant, The Ohio State University, and Universidad Veracruzana.

\section{ORCID}

Liliana Cortés-Ortiz (D) http://orcid.org/0000-0002-1197-6362

\section{REFERENCES}

Arnold, M. L. (2006). Evolution through genetic exchange. Oxford, UK: Oxford University Press.

Arnold, M. L., \& Meyer, A. (2006). Natural hybridization in primates: One evolutionary mechanism. Zoology, 109, 261-276. https://doi.org/10. 1016/j.zool.2006.03.006

Arroyo-Rdríguez, V., \& Dias, P. A. D. (2010). Effects of habitat fragmentation and disturbance on howler monkeys: A review. American Journal of Primatology, 72, 1-16. https://doi.org/10.1002/ ajp.20753

Bergman, T. J., \& Beehner, J. C. (2004). The social system of a hybrid baboon group (Papio hamadryas anubis x P. h. hamadryas). International Journal of Primatology, 25, 1313-1330. https://doi.org/10.1023/ B:IJOP.0000

Bergman, T. J., Cortés-Ortiz, L., Dias, P. A. D., Ho, L., Adams, D., CanalesEspinosa, D., \& Kitchen, D. M. (2016). Striking differences in the loud calls of howler monkey sister species (Alouatta pigra and A. palliata). American Journal of Primatology, 78, 755-766. https://doi.org/10.1002/ ajp.22539

Cortés-Ortiz, L., Duda, T. F., Canales-Espinosa, D., García-Orduña, F., Rodríguez-Luna, E., \& Bermingham, E. (2007). Hybridization in largebodied New World primates. Genetics, 176, 2421-2425. https://doi. org/10.1534/genetics.107.074278

Cortés-Ortiz, L., Nidiffer, M. D., Hermida-Lagunes, J., García-Orduña, F., Kitchen, D. M., Bergman, T., Dias, P. A. D., \& Canales-Espinosa, D. (2019). Reduced introgression of sex chromosome markers in the Mexican howler monkey (Alouatta palliata x A. pigra) hybrid zone. International Journal of Primatology, 40, 114-131. https://doi.org/10. 1007/s10764-018-0056-4

Cortés-Ortiz, L., Roos, C., \& Zinner, D. (2019). Introduction to special issue on primate hybridization and hybrid zones. International Journal of Primatology, 40, 1-8. https://doi.org/10.1007/s10764-019-00076-z

Grant, P. R., \& Grant, B. R. (1992). Hybridization of bird species. Science, 256, 193-197. https://doi.org/10.1126/science.256.5054.193

Green, R. E., Krause, J., Briggs, A. W., Maricic, T., Stenzel, U., Kircher, M., \& Pääbo, S. (2010). A draft sequence of the neandertal genome. Science, 328, 710-722. https://doi.org/10.1126/science.1188021

Ho, L., Cortés-Ortiz, L., Dias, P. A., Canales-Espinosa, D., Kitchen, D. M., \& Bergman, T. J. (2014). Effect of ancestry on behavioral variation in two species of howler monkeys (Alouatta pigra and A. palliata) and their hybrids. American Journal of Primatology, 76, 855-867. https:// doi.org/10.1002/ajp.22273

Kelaita, M., Dias, P.A.D., Aguilar-Cucurachi, M.S., Canales-Espinosa, D., \& Cortés-Ortiz, L. (2011). Impact of intra-sexual selection on sexual dimorphism and testes size in the Mexican howler monkeys Alouatta 
palliata and A. pigra. America Journal of Physical Anthropology, 146(2), 179-187.

Kelaita, M. A., \& Cortés-Ortiz, L. (2013). Morphological variation of genetically confirmed Alouatta pigra $\times$ A. palliata hybrids from a natural hybrid zone in Tabasco, Mexico. American Journal of Physical Anthropology, 150, 223-234. https://doi.org/10.1002/ajpa.22196

Kitchen, D. M. (2004). Alpha male black howler monkey responses to loud calls: Effect of numeric odds, male companion behaviour, and reproductive investment. Animal Behaviour, 67, 125-139. https://doi. org/10.1016/j.anbehav.2003.03.007

Kitchen, D. M., Bergman, T. J., Dias, P. A. D., Ho, L., Canales-Espinosa, D., \& Cortés-Ortiz, L. (2019). Temporal but not acoustic plasticity in hybrid howler monkey (Alouatta palliata $\times$ A. pigra) loud calls. International Journal of Primatology, 40, 132-152. https://doi.org/10.1007/s10764-017-0004-8

Kitchen, D. M., Cortés-Ortiz, L., Dias, P. A. D., Canales-Espinosa, D., \& Bergman, T. J. (2018). Alouatta pigra males ignore A. palliata loud calls: A case of failed rival recognition? American Journal of Physical Anthropology, 166, 433-441. https://doi.org/10.1002/ajpa.23443

Kitchen, D. M., Seyfarth, R. M., Fischer, J., \& Cheney, D. L. (2003). Loud calls as indicators of dominance in male baboons (Papio cynocephalus ursinus). Behavioral Ecology and Sociobiology, 53, 374-384. https://doi. org/10.1007/s00265-003-0588-1

Mallet, J. (2005). Hybridization as an invasion of the genome. Trends in Ecology and Evolution, 20, 229-237. https://doi.org/10.1016/j.tree. 2005.02.010
Reich, D., Green, R. E., Kircher, M., Krause, J., Patterson, N., Durand, E. Y., \& Pääbo, S. (2010). Genetic history of an archaic hominin group from Denisova Cave in Siberia. Nature, 468, 1053-1060. https://doi.org/10. 1038/nature09710

Sankararaman, S., Mallick, S., Dannemann, M., Prüfer, K., Kelso, J., Pääbo, S., \& Reich, D. (2014). The genomic landscape of Neanderthal ancestry in present-day humans. Nature, 507, 354-357. https://doi.org/10. 1038/nature12961

Smith, J. D. (1970). The systematic status of the black howler monkey, Alouatta pigra Lawrence. Journal of Mammalogy, 51, 358-369. https:// doi.org/10.2307/1378486

Zinner, D., Arnold, M. L., \& Roos, C. (2011). The strange blood: Natural hybridization in primates. Evolutionary Anthropology, 20, 96-103. https://doi.org/10.1002/evan.20301

How to cite this article: Cortés-Ortiz L, Espinosa DC, Dias PD, Bergman T, Kitchen DM. From Mexico to Michigan and back: An international collaboration investigating primate behavior, ecology, and evolution from multiple perspectives.

Am J Primatol. 2019;81:e22992.

https://doi.org/10.1002/ajp.22992 